\title{
MR Imaging in Traumatic Distal Biceps Brachii Tendon Ruptures
}

Labata Alonso Álvaro*, Pérez Rivarés Carmen and Ramos Gadea Anna

Radiology Technologist Reference MRI, Institut de Diagnòstic per lalmatge IDI Hospital Universitari Joan XXIII and Mutua Asepeyo, Tarragona, Spain

*Corresponding author: Labata Alonso Álvaro, Radiology Technologist Reference MRI, Institut de Diagnòstic per la Imatge, Hospital Universitari Joan XXIII, Tarragona, Spain, E-mail: alvarolabata@gmail.com

Received date: August 09, 2018; Accepted date: October 01, 2018; Published date: October 08, 2018

Copyright: @2018 Álvaro LA, et al. This is an open-access article distributed under the terms of the Creative Commons Attribution License, which permits unrestricted use, distribution, and reproduction in any medium, provided the original author and source are credited.

\begin{abstract}
The biceps muscle is inserted in its distal part in the bicipital tuberosity of the radius through the lower biceps tendon. If the tendon is subjected to excessive stress, it may break completely or partially. Distal biceps tendon rupture is very rare, when it occurs it requires surgery in most cases.
\end{abstract}

Keywords: Bicipital tuberosity; Distal biceps; Distal tendon

\section{Methodology}

We present the case of a patient with suspected rupture of the distal biceps tendon while traveling in a boat. After the clinical examination, the doctor requested an MRI [1-3]. We performed the study with General Electric Signa HDX 1.5T MR, knee coil, positioning of the affected arm of the patient in "Superman" mode, in extension and the hand resting on the fifth metacarpus and the thumb up. Almost always we get that the affected arm is in the isocenter of the magnet, thus we get a better field homogeneity. The exploration, if the patient does not move, usually lasts about 40 minutes. We have to take great care of the patient's comfort, with the help of cushions and pillows. We also provide some blankets to cover them due to the low temperatures of the magnet room.

Our usual protocol always starts with the patient in the "Superman" position because of the 100 MRI [4,5] elbow scans done every year, only $2 \%$ are due to rupture of the distal tendon of the brachial biceps, for this reason we are more familiar with the anatomy of the elbow in this position that does not place the patient in position FABS directly. We will make sequences (useful mainly for ligaments and tendons) T2 and STIR (useful for soft tissue edema) in the three planes of the space with a thickness of between 3 and $4 \mathrm{~mm}$ and a distance between cuts of not more than $1.5 \mathrm{~mm}$ (Figures 1-3).
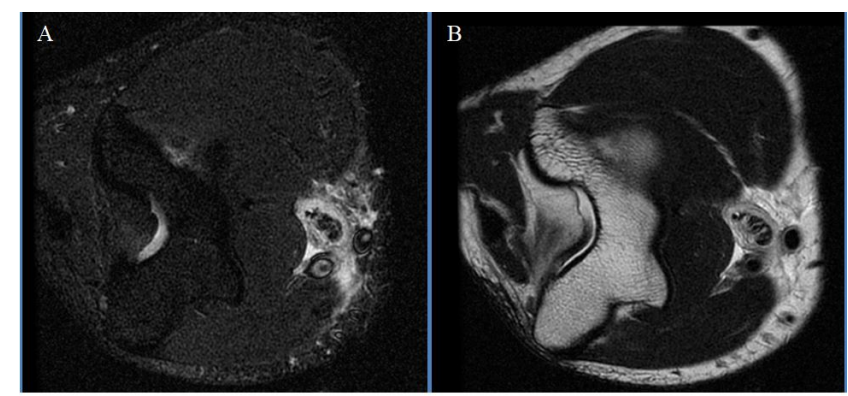

Figure 1: (A) Axial STIR, (B) Axial T2.
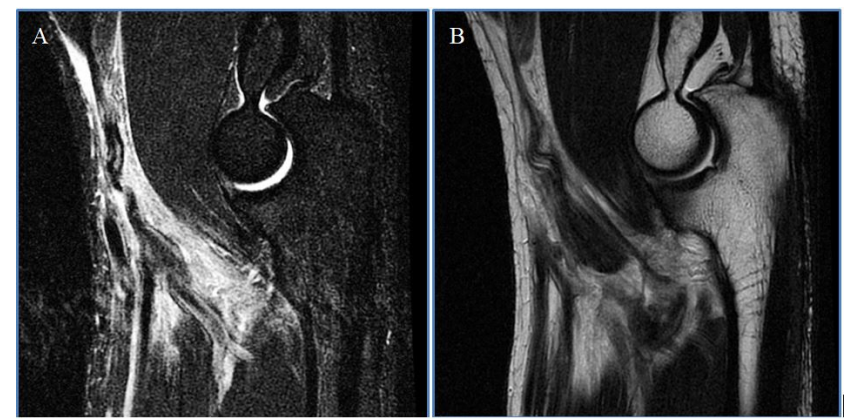

Figure 2: (A) Sagittal STIR, (B) Sagittal T2.

The traditional axial image of the distal tendon of the biceps brachii is also a valuable means of evaluating this structure. In addition to providing a short axis image of the tendon, the axial series provides a familiar view of important associated structures, such as the median and radial nerves in the elbow.

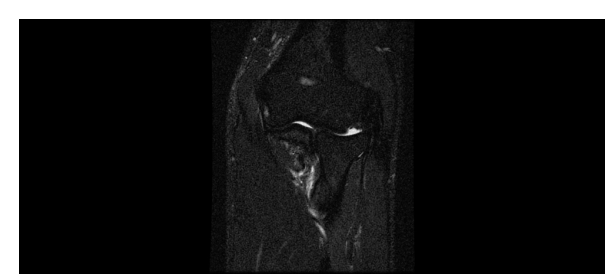

Figure 3: Cor STIR.

Then we made an STIR sequence with specific guidance of the distal biceps tendon in the following way. Slice thickness $3.5 \mathrm{~mm}$ and a distance between slices not more than $1 \mathrm{~mm}$, (Figure 4). 
Citation: ÁlvaroLA, Carmen PR, Anna RG (2018) MR Imaging in Traumatic Distal Biceps Brachii Tendon Ruptures. J Nucl Med Radiat Ther 9:

Page 2 of 2

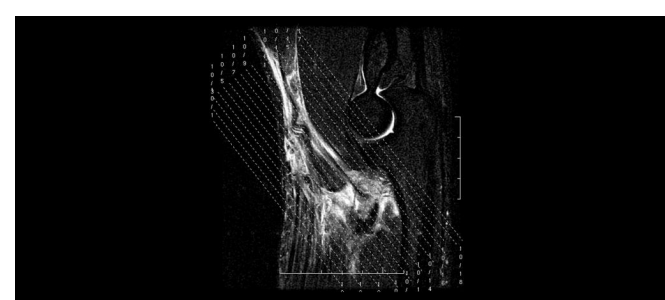

Figure 4: Sagittal short T1 inversion recovery (STIR) imaging on which the oblicue coronal slices are reflected.

The oblique coronal resulting images that are shown below, following the direction of the tendon injury with slice thickness 3.5 $\mathrm{mm}$ and a distance between slices not more than $1 \mathrm{~mm}$ (Figure 5).
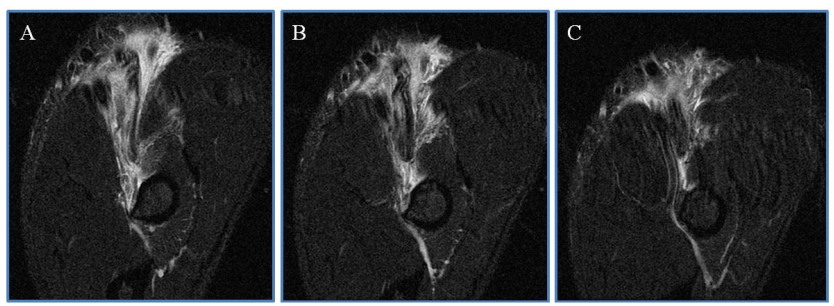

Figure 5: (A-C) Three consecutive oblique coronal STIR images, thickness $3.5 \mathrm{~mm}$ and distance between images not more than 1 $\mathrm{mm}$ demonstrate the rupture of the distal brachii tendon of the biceps.

Then, we will see accurately the distance of the tendon broken respect the tuberosity. If the STIR sequence with double obliquity is not conclusive, we need to reposition the patient in FABS [6,7], Flexed Abducted Supinated position (bending elbow, abduction shoulder, arm Supination) and so try to get a good longitudinal view full tendon.

\section{Results}

The set of images in different planes of space showed the clinical suspicion of distal complete rupture of tendon of brachial biceps [8], showing a slight retraction proximally, identified the altered signal break end, of morphology undulating and located approximately 15 $\mathrm{mm}$ proximally to the bicipital tuberosity of the radius. The rest of muscle structures, tendons and bone are within normal limits. The treatment was early surgical repair, using an anterior approach, with a suture anchor to reattach the tendon to the radial tuberosity.

\section{Discusion}

As we have explained previously, the usual practice in our work when we do an MRI requested by the orthopedic surgeon to study the rupture of the distal biceps tendon $[9,10]$ is to perform the sequences with the patient lying in "Superman" mode, since we are more used to see the anatomical structures of the elbow joint in this way, therefore, we are easier to interpret, as well we have trained our eyesight. What we do is complete the "FABS" mode of study when we cannot discern if there is a rupture of the distal tendon of the biceps in "Superman" mode, but this fact in our case rarely occurs.

\section{References}

1. Hayter CL, Giuffre BM (2009) Overuse and traumatic injuries of the elbow. Magn Reson Imaging Clin N Am 17: 617-638

2. Fritz RC (1999) MR imaging of sports injuries of the elbow. Magn Reson Imaging Clin N Am 7: 51-72

3. Thornton R, Riley GM, Steinbach LS (2003) Magnetic resonance imaging of sports injuries of the elbow. Top Magn Reson Imaging 14: 69-86.

4. Quaia E, Bazzocchi M, Zuiani C, Moroldo ML (1998) Magnetic resonance of the elbow: Technique optimization and definitions of normal anatomical features and their variations. Radiol Med 95: 7-15.

5. Fritz RC, Steinbach LS (1996) Magnetic resonance imaging of the musculoskeletal system: Part The elbow. Clin Orthop Relat Res 324: 321-339.

6. Giuffrè BM1, Moss MJ (2004) Optimal positioning for MRI of the distal biceps brachii tendon: flexed abducted supinated view. AJR Am J Roentgenol 182: 944-946.

7. Fitzgerald SW1, Curry DR, Erickson SJ, Quinn SF, Friedman H (1994) Distal biceps tendon injury: MR imaging diagnosis. Radiology 191: 203-206.

8. Chew ML, Giuffrè BM (2005) Disorders of the distal biceps brachii tendon. Radiographics 25: 1227-1237.

9. Festa A, Mulieri PJ, Newman JS, Spitz DJ, Leslie BM (2010) Effectiveness of magnetic resonance imaging in detecting partial and complete distal biceps tendon rupture. J Hand Surg Am 35: 77-83.

10. Williams BD, Schweitzer ME, Weishaupt D, Lerman J, Rubenstein DL, et al. (2001) Partial tears of the distal biceps tendon: MR appearance and associated clinical findings. Skeletal Radiol 30: 560-504. 